УДК 517.518.232

\author{
O. M. BUHRII
}

\title{
ON $\varepsilon$-FRIEDRICHS INEQUALITIES AND ITS APPLICATION
}

O. M. Buhrii. On $\varepsilon$-Friedrichs inequalities and its application, Mat. Stud. 51 (2019), 19-24.

Let $n \in \mathbb{N}$ be a fixed number, $\Omega$ be a bounded domain in $\mathbb{R}^{n}, L^{2}(\Omega), L^{\infty}(\Omega)$ be the Lebesgue spaces, $H^{1}(\Omega)$ and $H_{0}^{1}(\Omega)$ be the Sobolev spaces,

$$
\Pi_{\ell}(\alpha):=\otimes_{k=1}^{n}\left(\alpha_{k} ; \alpha_{k}+\ell\right), \quad \alpha=\left(\alpha_{1}, \ldots, \alpha_{n}\right) \in \mathbb{R}^{n}, \quad \ell>0 .
$$

There are proved the following assertions about $\varepsilon$-Friedrichs inequality (Theorem 1) in the space $H^{1}(\Omega)$ (Theorem 1) and $H_{0}^{1}(\Omega)$ (Theorem 2) : for every $\varepsilon>0$ there exist $N_{\varepsilon} \in \mathbb{N}$ and $\omega_{1}, \ldots, \omega_{N_{\varepsilon}} \in L^{\infty}(\Omega)$ such that the inequality

$$
\int_{\Omega}|v(x)|^{2} d x \leq \varepsilon \int_{\Omega}|\nabla v(x)|^{2} d x+\sum_{j=1}^{N_{\varepsilon}}\left(\int_{\Omega} v(x) \omega_{j}(x) d x\right)^{2}
$$

holds for every $v \in H^{1}(\Omega)$ (Theorem 1) and $v \in H_{0}^{1}(\Omega)$ (Theorem 2), where $\Omega$ is a bounded domain in $\mathbb{R}^{n}$ for which there exist numbers $\ell>0, m \in \mathbb{N}$, and $\alpha^{1}, \ldots, \alpha^{m} \in \mathbb{R}^{n}$ satisfying the following conditions

1) $\bar{\Omega}=\overline{\Pi_{\ell}\left(\alpha^{1}\right)} \cup \ldots \cup \overline{\Pi_{\ell}\left(\alpha^{m}\right)}$;

2) for every $i, j \in\{1, \ldots, m\}$ with $i \neq j$ we obtain: $\Pi_{\ell}\left(\alpha^{i}\right) \cap \Pi_{\ell}\left(\alpha^{j}\right)=\varnothing$.

Let $n \in \mathbb{N}$ be a fixed number, $\Omega$ be a bounded domain in $\mathbb{R}^{n}, L^{2}(\Omega), L^{\infty}(\Omega)$ be the Lebesgue spaces (see [1, p. 618]), $H^{1}(\Omega)$ and $H_{0}^{1}(\Omega)$ be the Sobolev spaces (see [1, p. 245$246]), H$ be a Hilbert space, $C([0, T] ; H)$ be the space of the $H$-valued continuous functions defined on $[0, T]$ (see [2, p. 147]), $L^{2}(0, T ; H)$ be the Lebesgue-Bochner space (see [2, p. 155]). We define

$$
C_{\text {weak }}([0, T] ; H):=\left\{u:[0, T] \rightarrow H \quad \mid \forall z \in H \quad(u(\cdot), z)_{H} \in C([0, T])\right\} .
$$

Note that $C([0, T] ; H) \varsubsetneqq C_{\text {weak }}([0, T] ; H)$ (see [2, p. 147] for more details).

We recall (see Lemma 1.26 [2, Chapter 2, §1]) the standard Friedrichs inequality:

$$
\int_{\Omega}|v(x)|^{2} d x \leq M_{\Omega} \int_{\Omega}|\nabla v(x)|^{2} d x, \quad v \in H_{0}^{1}(\Omega),
$$

where $\nabla v:=\left(v_{x_{1}}, \ldots, v_{x_{n}}\right)$. Notice that the constant $M_{\Omega}>0$ depends on $\Omega$ and does not depend on $v$. The various generalizations of formula (1) are considered in [3], [4], [5], etc.

In the paper, we consider some modification of (1). We denote

$$
\Pi_{\ell}(\alpha):=\left(\alpha_{1} ; \alpha_{1}+\ell\right) \times\left(\alpha_{2} ; \alpha_{2}+\ell\right) \times \ldots \times\left(\alpha_{n} ; \alpha_{n}+\ell\right), \quad \alpha=\left(\alpha_{1}, \ldots, \alpha_{n}\right) \in \mathbb{R}^{n}, \quad \ell>0 .
$$

2010 Mathematics Subject Classification: 26D10, 35A23, 46E35.

Keywords: the Friedrichs inequality, the Sobolev space.

doi: $10.15330 / \mathrm{ms} .51 .1 .19-24$

(C) O. M. Buhrii, 2019 
Clearly, $\Pi_{\ell}(\alpha)$ is a cube in $\mathbb{R}^{n}$ with the volume $\ell^{n}$. Let us define the set $\Pi\left(\mathbb{R}^{n}\right)$ as follows. For every $\Omega \in \Pi\left(\mathbb{R}^{n}\right)$ we have that $\Omega$ is a bounded domain in $\mathbb{R}^{n}$ and there exist numbers $\ell>0, m \in \mathbb{N}$, and $\alpha^{1}, \ldots, \alpha^{m} \in \mathbb{R}^{n}$ such that the following conditions hold:

1) $\bar{\Omega}=\overline{\Pi_{\ell}\left(\alpha^{1}\right)} \cup \ldots \cup \overline{\Pi_{\ell}\left(\alpha^{m}\right)}$;

2) for every $i, j \in\{1, \ldots, m\}$ such that $i \neq j$ we obtain: $\Pi_{\ell}\left(\alpha^{i}\right) \cap \Pi_{\ell}\left(\alpha^{j}\right)=\varnothing$. The main results of the paper are contained in the next propositions.

Theorem 1 ( $\varepsilon$-Friedrichs inequality for the functions from $\left.H^{1}(\Omega)\right)$. If $\Omega \in \Pi\left(\mathbb{R}^{n}\right)$, then for every $\varepsilon>0$ there exist $N_{\varepsilon} \in \mathbb{N}$ and $\omega_{1}, \ldots, \omega_{N_{\varepsilon}} \in L^{\infty}(\Omega)$ such that the inequality

$$
\int_{\Omega}|v(x)|^{2} d x \leq \varepsilon \int_{\Omega}|\nabla v(x)|^{2} d x+\sum_{j=1}^{N_{\varepsilon}}\left(\int_{\Omega} v(x) \omega_{j}(x) d x\right)^{2}
$$

holds for every $v \in H^{1}(\Omega)$.

Theorem 2 ( $\varepsilon$-Friedrichs inequality for functions from $H_{0}^{1}(\Omega)$ ). If $\Omega$ is a bounded domain in $\mathbb{R}^{n}$, then for every $\varepsilon>0$ there exist $N_{\varepsilon} \in \mathbb{N}$ and $\omega_{1}, \ldots, \omega_{N_{\varepsilon}} \in L^{\infty}(\Omega)$ such that inequality (3) holds for every $v \in H_{0}^{1}(\Omega)$.

Corollary 1. Suppose that $\Omega$ is taken from either Theorem 1 or Theorem 2, $T>0$ is given number, $H:=L^{2}(\Omega), V:=H^{1}(\Omega)$ if $\Omega \in \Pi\left(\mathbb{R}^{n}\right), V:=H_{0}^{1}(\Omega)$ if $\Omega$ satisfies the conditions of Theorem 2, and $u^{m} \underset{m \rightarrow \infty}{\longrightarrow} u$ in $C_{\text {weak }}([0, T] ; H)$. Then the following statements are fulfilled:

(i) if $u^{m} \underset{m \rightarrow \infty}{\longrightarrow} u$ slowly in $L^{2}(0, T ; V)$, then $u^{m} \underset{m \rightarrow \infty}{\longrightarrow} u$ strongly in $L^{2}(0, T ; H)$;

(ii) if $u^{m} \underset{m \rightarrow \infty}{\longrightarrow} u *$-slowly in $L^{\infty}(0, T ; V)$, then $u^{m} \underset{m \rightarrow \infty}{\longrightarrow} u$ in $C([0, T] ; H)$.

Note that the case $n=2$ of Theorem 2 is considered in [6, p. 553] and [7, p. 172]. We give the proof of Theorem 2 in case $n \neq 2$ for convenience. As we know the $\varepsilon$-Friedrichs inequalities for functions from $H^{1}(\Omega)$ is not studied yet.

Let us prove the main results. Clearly,

$$
\left(a_{1}+\ldots+a_{n}\right)^{2}=\left(1 \cdot a_{1}+\ldots+1 \cdot a_{n}\right)^{2} \leq\left(\sum_{i=1}^{n} 1^{2}\right)\left(\sum_{i=1}^{n} a_{i}^{2}\right) \leq n\left(a_{1}^{2}+\ldots+a_{n}^{2}\right) .
$$

The following lemma is needed for the sequel.

Lemma 1 (Poincaré's inequality for cube in $\left.\mathbb{R}^{n}\right)$. If $n \in \mathbb{N}, \alpha=\left(\alpha_{1}, \ldots, \alpha_{n}\right) \in \mathbb{R}^{n}, \ell>0$, and $G=\Pi_{\ell}(\alpha)$, where $\Pi_{\ell}(\alpha)$ is chosen from (2), then for every $v \in H^{1}(G)$ we have

$$
\int_{G}|v(\xi)|^{2} d \xi \leq \frac{n}{2} \ell^{2} \int_{G}|\nabla v(\xi)|^{2} d \xi+\frac{1}{\ell^{n}}\left(\int_{G} v(\xi) d \xi\right)^{2} .
$$

Proof. Note that the case $n=2$ is considered in [6, p. 552]. Suppose that $n \in \mathbb{N} \backslash\{2\}$. For simplicity, we prove Lemma 1 only if $n=3$ and $\alpha_{1}=\ldots=\alpha_{n}=0$.

Let $n=3, \ell>0$, and $G=(0 ; \ell)^{n}$. Take arbitrary points $\xi_{1}=\left(x_{1}, y_{1}, z_{1}\right) \in G$ and $\xi_{2}=\left(x_{2}, y_{2}, z_{2}\right) \in G$. Then, for every $v \in H^{1}(G)$, we have

$$
v\left(x_{2}, y_{2}, z_{2}\right)-v\left(x_{1}, y_{1}, z_{1}\right)=v\left(x_{2}, y_{2}, z_{2}\right)-v\left(x_{1}, y_{2}, z_{2}\right)+v\left(x_{1}, y_{2}, z_{2}\right)-v\left(x_{1}, y_{1}, z_{2}\right)+
$$




$$
+v\left(x_{1}, y_{1}, z_{2}\right)-v\left(x_{1}, y_{1}, z_{1}\right)=\int_{x_{1}}^{x_{2}} v_{x}\left(x, y_{2}, z_{2}\right) d x+\int_{y_{1}}^{y_{2}} v_{y}\left(x_{1}, y, z_{2}\right) d y+\int_{z_{1}}^{z_{2}} v_{z}\left(x_{1}, y_{1}, z\right) d z .
$$

Squaring this equality and using inequality (4) with $n=3$, the Cauchy-Bunyakowski-Schwarz inequality, and the conditions

$$
\left|x_{2}-x_{1}\right| \leq \ell, \quad\left|y_{2}-y_{1}\right| \leq \ell, \quad\left|z_{2}-z_{1}\right| \leq \ell
$$

we obtain

$$
\begin{gathered}
\left|v\left(x_{2}, y_{2}, z_{2}\right)-v\left(x_{1}, y_{1}, z_{1}\right)\right|^{2} \leq \\
\leq n\left\{\left(\int_{x_{1}}^{x_{2}} v_{x}\left(x, y_{2}, z_{2}\right) d x\right)^{2}+\left(\int_{y_{1}}^{y_{2}} v_{y}\left(x_{1}, y, z_{2}\right) d y\right)^{2}+\left(\int_{z_{1}}^{z_{2}} v_{z}\left(x_{1}, y_{1}, z\right) d z\right)^{2}\right\} \leq \\
\leq n \ell\left\{\int_{0}^{\ell}\left|v_{x}\left(x, y_{2}, z_{2}\right)\right|^{2} d x+\int_{0}^{\ell}\left|v_{y}\left(x_{1}, y, z_{2}\right)\right|^{2} d y+\int_{0}^{\ell}\left|v_{z}\left(x_{1}, y_{1}, z\right)\right|^{2} d z\right\} .
\end{gathered}
$$

Integrating this inequality in $\left(x_{1}, y_{1}, z_{1}\right),\left(x_{2}, y_{2}, z_{2}\right) \in G$, we deduce

$$
\begin{gathered}
J \leq n \ell \int_{G} d x_{1} d y_{1} d z_{1} \int_{G}\left\{\int_{0}^{\ell}\left|v_{x}\left(x, y_{2}, z_{2}\right)\right|^{2} d x+\int_{0}^{\ell}\left|v_{y}\left(x_{1}, y, z_{2}\right)\right|^{2} d y+\right. \\
\left.+\int_{0}^{\ell}\left|v_{z}\left(x_{1}, y_{1}, z\right)\right|^{2} d z\right\} d x_{2} d y_{2} d z_{2}=n \ell \cdot \ell^{n+1} \int_{G}\left[\left|v_{x}(x, y, z)\right|^{2}+\left|v_{y}(x, y, z)\right|^{2}+\right. \\
\left.+\left|v_{z}(x, y, z)\right|^{2}\right] d x d y d z=n \ell^{n+2} \int_{G}|\nabla v(x, y, z)|^{2} d x d y d z
\end{gathered}
$$

where

$$
J:=\int_{G} d x_{1} d y_{1} d z_{1} \int_{G}\left|v\left(x_{2}, y_{2}, z_{2}\right)-v\left(x_{1}, y_{1}, z_{1}\right)\right|^{2} d x_{2} d y_{2} d z_{2}
$$

Clearly, (8) implies

$$
\begin{gathered}
J=\int_{G} d x_{1} d y_{1} d z_{1} \int_{G}\left[\left|v\left(x_{2}, y_{2}, z_{2}\right)\right|^{2}+\left|v\left(x_{1}, y_{1}, z_{1}\right)\right|^{2}-2 v\left(x_{2}, y_{2}, z_{2}\right) v\left(x_{1}, y_{1}, z_{1}\right)\right] d x_{2} d y_{2} d z_{2}= \\
=2 \ell^{n} \int_{G}|v(x, y, z)|^{2} d x d y d z-2\left(\int_{G} v\left(x_{2}, y_{2}, z_{2}\right) d x_{2} d y_{2} d z_{2}\right) \cdot\left(\int_{G} v\left(x_{1}, y_{1}, z_{1}\right) d x_{1} d y_{1} d z_{1}\right)= \\
=2 \ell^{n} \int_{G}|v(x, y, z)|^{2} d x d y d z-2\left(\int_{G} v(x, y, z) d x d y d z\right)^{2} \cdot
\end{gathered}
$$

Then from (7) we obtain

$$
2 \ell^{n} \int_{G}|v|^{2} d x d y d z-2\left(\int_{G} v d x d y d z\right)^{2} \leq n \ell^{n+2} \int_{G}|\nabla v|^{2} d x d y d z,
$$

Therefore, (5) holds and Lemma 1 is proved. 
Proof of Theorem 1. Since $\Omega \in \Pi\left(\mathbb{R}^{n}\right)$, we have $L>0, m \in \mathbb{N}$, and $\alpha^{1}, \ldots, \alpha^{m} \in \mathbb{R}^{n}$ such that $\bar{\Omega}=\overline{\Pi_{L}\left(\alpha^{1}\right)} \cup \ldots \cup \overline{\Pi_{L}\left(\alpha^{m}\right)}$ and $\Pi_{L}\left(\alpha^{i}\right) \cap \Pi_{L}\left(\alpha^{j}\right)=\varnothing$ if $i \neq j$.

Take $k \in \mathbb{N}$ and put $N=k^{n}$. For every $j \in\{1, \ldots, m\}$ we partition every cube $\Pi_{L}\left(\alpha^{j}\right)$ into cubes $Q_{1}^{k, j}, Q_{2}^{k, j}, \ldots, Q_{N}^{k, j}$ such that every cube $Q_{\mu}^{k, j}$ has its side with length $\ell=\frac{L}{k}$.

Let us consider an arbitrary $v \in H^{1}(\Omega)$. For every $j \in\{1, \ldots, m\}$ and $\mu \in\{1, \ldots, N\}$, we use Poincaré's inequality (5) with $G=Q_{\mu}^{k, j}$. Summing these inequalities, we get

$$
J_{1} \leq J_{2}+J_{3}
$$

where (for the sake of convenience we replace $\xi$ by $x$ )

$$
\begin{gathered}
J_{1}:=\sum_{j=1}^{m} \sum_{\mu=1}^{N} \int_{Q_{\mu}^{k, j}}|v(x)|^{2} d x, \quad J_{2}:=\sum_{j=1}^{m} \sum_{\mu=1}^{N} \frac{n}{2} \ell^{2} \int_{Q_{\mu}^{k, j}}|\nabla v(x)|^{2} d x, \\
J_{3}:=\sum_{j=1}^{m} \sum_{\mu=1}^{N} \frac{1}{\ell^{n}}\left(\int_{Q_{\mu}^{k, j}} v(x) d x\right)^{2} .
\end{gathered}
$$

Clearly, $J_{1}=\int_{\Omega}|v|^{2} d x, \quad J_{2}=\frac{n}{2} \ell^{2} \int_{\Omega}|\nabla v|^{2} d x$. If we take

$$
\chi_{\mu}^{k, j}(x):=\left\{\begin{array}{ll}
1, & x \in Q_{\mu}^{k, j}, \\
0, & x \in \mathbb{R}^{n} \backslash Q_{\mu}^{k, j},
\end{array} \quad j=\overline{1, m}, \quad \mu=\overline{1, N},\right.
$$

then

$$
J_{3}=\sum_{j=1}^{m} \sum_{\mu=1}^{N} \frac{1}{\ell^{n}}\left(\int_{\Omega} v(x) \chi_{\mu}^{k, j}(x) d x\right)^{2}
$$

Thus, (10) yields that

$$
\int_{\Omega}|v|^{2} d x \leq \frac{n L^{2}}{2} \cdot \frac{1}{k^{2}} \int_{\Omega}|\nabla v|^{2} d x+\frac{1}{L^{n}} \cdot k^{n} \sum_{j=1}^{m} \sum_{\mu=1}^{k^{n}}\left(\int_{\Omega} v(x) \chi_{\mu}^{k, j}(x) d x\right)^{2}, \quad k \in \mathbb{N} .
$$

Finally, for every $\varepsilon>0$ we choose $k \in \mathbb{N}$ such that $\frac{n L^{2}}{2} \cdot \frac{1}{k^{2}} \leq \varepsilon$. Then, from (11) we obtain (3) and Theorem 1 is proved.

Proof of Theorem 2. Note that the case $n=2$ is considered in [6, p. 542, 543, 553]. Suppose that $n \in \mathbb{N} \backslash\{2\}$ and modify the proof of Theorem 1 .

Let $Q$ be a cube in $\mathbb{R}^{n}$ such that $\Omega \subset Q$ and the length of the side of $Q$ equals $L>0$. Take $k \in \mathbb{N}$ and put $N=k^{n}$. We partition the cube $Q$ into the cubes $Q_{1}^{k}, Q_{2}^{k}, \ldots, Q_{N}^{k}$ such that every cube $Q_{\mu}^{k}$ has its side with length $\ell=\frac{L}{k}$.

We take an arbitrary $v \in H_{0}^{1}(\Omega)$. Assume that the function $v$ equals zero outside $\Omega$. For every $\mu \in\{1, \ldots, N\}$, we use Poincaré's inequality (5) with $G=Q_{\mu}^{k}$. Summing these inequalities from 1 to $N$, we get

$$
I_{1} \leq I_{2}+I_{3}
$$

where (for the sake of convenience we replace $\xi$ by $x$ again)

$$
I_{1}:=\sum_{\mu=1}^{N} \int_{Q_{\mu}^{k}}|v(x)|^{2} d x, \quad I_{2}:=\sum_{\mu=1}^{N} \frac{n}{2} \ell^{2} \int_{Q_{\mu}^{k}}|\nabla v(x)|^{2} d x, \quad I_{3}:=\sum_{\mu=1}^{N} \frac{1}{\ell^{n}}\left(\int_{Q_{\mu}^{k}} v(x) d x\right)^{2} .
$$


Clearly,

$$
I_{1}=\int_{Q}|v|^{2} d x=\int_{\Omega}|v|^{2} d x, \quad I_{2}=\frac{n}{2} \ell^{2} \int_{Q}|\nabla v|^{2} d x=\frac{n}{2} \ell^{2} \int_{\Omega}|\nabla v|^{2} d x .
$$

If we choose

$$
\chi_{\mu}^{k}(x)= \begin{cases}1, & x \in Q_{\mu}^{k}, \\ 0, & x \in \mathbb{R}^{n} \backslash Q_{\mu}^{k}, \quad \mu=\overline{1, N},\end{cases}
$$

then

$$
I_{3}=\sum_{\mu=1}^{N} \frac{1}{\ell^{n}}\left(\int_{Q} v(x) \chi_{\mu}^{k}(x) d x\right)^{2}=\sum_{\mu=1}^{N} \frac{1}{\ell^{n}}\left(\int_{\Omega} v(x) \chi_{\mu}^{k}(x) d x\right)^{2} .
$$

Thus, (12) yields that

$$
\int_{\Omega}|v|^{2} d x \leq \frac{n L^{2}}{2} \cdot \frac{1}{k^{2}} \int_{\Omega}|\nabla v|^{2} d x+\frac{1}{L^{n}} \cdot k^{n} \sum_{\mu=1}^{k^{n}}\left(\int_{\Omega} v(x) \chi_{\mu}^{k}(x) d x\right)^{2}, \quad k \in \mathbb{N} .
$$

Finally, for every $\varepsilon>0$ we choose $k \in \mathbb{N}$ such that $\frac{n L^{2}}{2} \cdot \frac{1}{k^{2}} \leq \varepsilon$. Then, from (13) we obtain (3).

Proof of Corollary 1. Suppose that $\left\{u^{m}\right\}_{m \in \mathbb{N}}$ satisfies condition (i) of Corollary 1. For a.e. $t \in(0, T)$, we use (3) with $v(x):=u^{m}(x, t)-u^{k}(x, t)$ for $x \in \Omega$. Integrating in $t$, we have

$\int_{Q_{0, T}}\left|u^{m}-u^{k}\right|^{2} d x d t \leq \varepsilon \int_{Q_{0, T}}\left|\nabla u^{m}-\nabla u^{k}\right|^{2} d x d t+\int_{0}^{T} \sum_{j=1}^{N_{\varepsilon}}\left(\int_{\Omega}\left[u^{m}(x, t)-u^{k}(x, t)\right] \omega_{j}(x) d x\right)^{2} d t$,

where $Q_{0, T}=\Omega \times(0, T)$ and $m, k \in \mathbb{N}$. The first integral on the right-hand side of this inequality does not exceed a fixed constant for any $m$ and $k$. Moreover, the last integral can be made arbitrary small for sufficiently large $m$ and $k$ because $\left(u^{m}(\cdot, t), \omega_{j}(\cdot)\right)_{L^{2}(\Omega)}$ uniform converges in $t$ as $m \rightarrow \infty$ (see [7, p. 173] for comparison). Therefore, the right-hand side of the inequality can be made arbitrary small for sufficiently large $m$ and $k$. Hence, the sequence $\left\{u^{m}\right\}_{m \in \mathbb{N}}$ converges strongly to $u$ in $L^{2}(0, T ; H)=L^{2}\left(Q_{0, T}\right)$.

If $\left\{u^{m}\right\}_{m \in \mathbb{N}}$ satisfies condition (ii), then the proof is similar.

\section{REFERENCES}

1. Evans L.C., Partial differential equations, Graduate Studies in Mathematics, Amer. Math. Soc., Providence, RI, 1998.

2. Gajewski H., Gröger K., Zacharias K., Nonlinear operator equations and operator differential equations, Mir, Moscow, 1978; translated from: Akademie-Verlag, Berlin, 1974.

3. Saranen J., On an inequality of Friedrichs, Mathematica Scandinavica, 51 (1983), №2, 310-322.

4. Brenner S.C., Poincaré-Friedrichs inequalities for piecewise H1 functions, SIAM J. Numer. Anal., 41 (2003), №1, 306-324. 
5. Andreev V.K., On inequalities of the Friedrichs type for combined domains, J. Siberian Federal Univ. Series Math. Phys., 2 (2009), №2, 146-157.

6. Courant R., Hilbert D., Methods of mathematical physics, V.2, Mir, Moscow, 1965.

7. Ladyzhenskaya O.A., The mathematical theory of viscous incompressible flow, GIFML, Moscow, 1961; English translation (second English edition): Gordon and Breach Science Publ., New York, London, Paris, Montreux, Tokyo, Melbourne, 1987.

Department of Mechanics and Mathematics, Ivan Franko National University of Lviv, Lviv, 79000, Ukraine

oleh.buhrii@lnu.edu.ua ol_buhrii@i.ua 